\title{
Renin-angiotensin system inhibitors and susceptibility to COVID-19 in patients with hypertension: a propensity score-matched cohort study in primary care
}

Shamil Haroon ${ }^{1 \dagger}$, Anuradhaa Subramanian ${ }^{1 \dagger}$, Jennifer Cooper ${ }^{1}$, Astha Anand ${ }^{1}$, Krishna Gokhale ${ }^{1}$, Nathan Byne ${ }^{2}$, Samir Dhalla ${ }^{3}$, Dionisio Acosta-Mena ${ }^{2}$, Thomas Taverner ${ }^{1}$, Kelvin Okoth¹, Jingya Wang ${ }^{1}$, Joht Singh Chandan ${ }^{1}$, Christopher Sainsbury ${ }^{1,4}$, Dawit Tefra Zemedikun', G. Neil Thomas', Dhruv Parekh ${ }^{5,6}$, Tom Marshall', Elizabeth Sapey ${ }^{5,6,7}$, Nicola J. Adderley ${ }^{1 *}$ (D) and Krishnarajah Nirantharakumar ${ }^{1,8,9}$

\begin{abstract}
Introduction: Renin-angiotensin system (RAS) inhibitors have been postulated to influence susceptibility to Severe Acute Respiratory Syndrome Coronavirus-2 (SARS-CoV-2). This study investigated whether there is an association between their prescription and the incidence of COVID-19 and all-cause mortality.

Methods: We conducted a propensity-score matched cohort study comparing the incidence of COVID-19 among patients with hypertension prescribed angiotensin-converting enzyme I (ACE) inhibitors or angiotensin II type-1 receptor blockers (ARBs) to those treated with calcium channel blockers (CCBs) in a large UK-based primary care database (The Health Improvement Network). We estimated crude incidence rates for confirmed/suspected COVID19 in each drug exposure group. We used Cox proportional hazards models to produce adjusted hazard ratios for COVID-19. We assessed all-cause mortality as a secondary outcome.
\end{abstract}

Results: The incidence rate of COVID-19 among users of ACE inhibitors and CCBs was 9.3 per 1000 person-years (83 of 18,895 users [0.44\%]) and 9.5 per 1000 person-years (85 of 18,895 [0.45\%]), respectively. The adjusted hazard ratio was 0.92 ( $95 \% \mathrm{Cl} 0.68$ to 1.26). The incidence rate among users of ARBs was 15.8 per 1000 person-years ( 79 out of 10,623 users [0.74\%]). The adjusted hazard ratio was 1.38 ( $95 \% \mathrm{Cl} 0.98$ to 1.95). There were no significant associations between use of RAS inhibitors and all-cause mortality.

\footnotetext{
* Correspondence: N.J.Adderley@bham.ac.uk

'Shamil Haroon and Anuradhaa Subramanian contributed equally to this work.

'Institute of Applied Health Research, University of Birmingham, Birmingham,

UK

Full list of author information is available at the end of the article
}

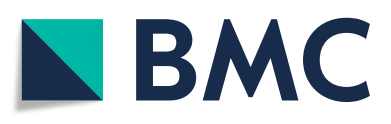

() The Author(s). 2021 Open Access This article is licensed under a Creative Commons Attribution 4.0 International License, which permits use, sharing, adaptation, distribution and reproduction in any medium or format, as long as you give appropriate credit to the original author(s) and the source, provide a link to the Creative Commons licence, and indicate if changes were made. The images or other third party material in this article are included in the article's Creative Commons licence, unless indicated otherwise in a credit line to the material. If material is not included in the article's Creative Commons licence and your intended use is not permitted by statutory regulation or exceeds the permitted use, you will need to obtain permission directly from the copyright holder. To view a copy of this licence, visit http://creativecommons.org/licenses/by/4.0/. The Creative Commons Public Domain Dedication waiver (http://creativecommons.org/publicdomain/zero/1.0/) applies to the data made available in this article, unless otherwise stated in a credit line to the data. 
(Continued from previous page)

Conclusion: Use of ACE inhibitors was not associated with the risk of COVID-19 whereas use of ARBs was associated with a statistically non-significant increase compared to the use of CCBs. However, no significant associations were observed between prescription of either ACE inhibitors or ARBs and all-cause mortality.

\section{Background}

A novel strain of coronavirus, Severe Acute Respiratory Syndrome Coronavirus-2 (SARS-CoV-2), was first detected in December 2019 in the district of Wuhan, China. This infection was found to cause a severe respiratory illness, termed COVID-19, which was associated with the development of acute respiratory distress syndrome (ARDS), particularly in older male adults, in those with obesity and comorbidities, and those from Black and Minority Ethnic backgrounds and low socioeconomic status [1]. The virus has caused a global pandemic that has crippled health systems and economies. As of 3rd January 2021, SARS-CoV-2 was estimated to have infected over 100 million people and caused over 2.2 million deaths [2].

Early on in the pandemic, a number of case series of patients with COVID-19 in China indicated a high prevalence of hypertension among those affected [3, 4]. Patients with hypertension appeared to have a threefold increase in the odds of mortality from COVID-19 compared to those without [5]. It is unclear whether this association was causal, and if so whether hypertension or antihypertensive drugs increased the risk of adverse outcomes from COVID-19. The renin angiotensin system (RAS) inhibitors, angiotensin-converting enzyme I (ACE) inhibitors and angiotensin II type-1 receptor blockers (ARBs), were specifically postulated to be involved in the pathogenesis of SARS-CoV-2 [6].

SARS-CoV-2 enters human cells using the angiotensinconverting enzyme 2 (ACE2) receptor, which is expressed in epithelial cells in human organs, including type II alveolar cells in the lungs as well as the cardiovascular system, kidneys, adrenal glands, brain, uterus and skin [7-9]. Experimental studies have suggested that use of ACE inhibitors and ARBs can upregulate ACE2 receptor expression in the cardiovascular and renal system [10]. Furthermore, the pathways within the renin-angiotensin system are complex and ACE inhibitors and ARBs may theoretically be protective because they increase concentrations of ACE2 and angiotensin [1-7], which have been shown to be protective in lung injury models [11].

The relationship between ACE inhibitors and ARBs and risk of COVID-19 need to be clarified as a large proportion of patients with hypertension, type 2 diabetes, heart failure, and chronic kidney disease, all of which are considered risk factors for COVID-19, are currently prescribed these drugs. In the absence of this evidence, it would not be appropriate to withdraw or switch these drugs as they are known to be cardioprotective and renoprotective. The Council on Hypertension of the European Society of Cardiology highlighted the lack of evidence supporting harmful effects of ACE inhibitors and ARBs in the context of COVID-19 early on in the pandemic [12]. Pharmacoepidemiological studies which account for confounding by indication bias are needed to address this evidence gap $[13,14]$.

Several epidemiological studies have attempted to investigate the association between RAS inhibitors and COVID-19 susceptibility. Some of the earliest published case-control studies, in Lombardy, Italy [15] and Denmark, found no significant association between use of RAS inhibitors and COVID-19 susceptibility, severity or mortality [16]. However, these studies included patients with a range of conditions that could also be indications for RAS inhibitors, introducing the potential for confounding by indication bias.

The ideal study design to answer this question would be a randomised controlled trial comparing RAS inhibitors to comparator drugs. However, such a trial would likely be unfeasible given the widespread use of these drugs in everyday clinical practice. However, one approach to disentangling the independent relationship between COVID-19 susceptibility and exposure to RASinhibitors is to study patients with hypertension while excluding those with other indicator conditions such as cardiovascular or chronic kidney disease, and comparing the incidence of COVID-19 among similar patients who have received a RAS inhibitor to those who have received an active comparator drug such as a calcium channel blocker (CCB). The objective of this study is to assess whether there is an independent association between the use of RAS-inhibitors and incidence of COVID-19 and all-cause mortality among patients with hypertension.

\section{Methods \\ Study design}

This is a propensity score-matched cohort study with active comparators, using routine primary care data.

\section{Data source}

We used data from The Health Improvement Network database. This is a large database of primary care records from UK general practices that use Vision electronic health record software. It includes data for approximately 14 million patients ( 2 million active patients) at over 640 
primary care practices. It includes coded data on patient demographics, diagnoses, primary care prescriptions, consultations and investigations. Practices contributing data to primary care as of 30th Jan 2020 (index date) were eligible for inclusion if they had shown acceptable mortality reporting and had the Vision system installed on or before 30th Jan 2019. Investigators had direct access to an up-todate extract of the data source.

\section{Study population}

Adults aged 18 years and older with a diagnosis of hypertension and who were registered with an eligible general practice before 30th Jan 2019 were included. In our primary analysis, we excluded patients with heart failure, diabetes, cardiovascular disease (ischaemic heart disease, transient ischaemic attack, stroke, and peripheral vascular disease), and chronic kidney disease (including patients with an estimated glomerular filtration rate $<30 / \mathrm{min} / 1.73 \mathrm{~m}^{2}$ ) as these comorbidities represented alternative indications for RAS inhibitors. We also excluded all patients who were pregnant during the index date or had contraindications to the exposure drugs (e.g. hypersensitivity to ACE inhibitors).

\section{Exposed and comparator groups}

We derived three main cohorts of patients defined by their prescription of one of three antihypertensive drugs - angiotensin-converting enzyme inhibitors (ACE inhibitors) and angiotensin II receptor blockers (ARBs), which were the two exposure drugs of interest, and calcium channel blockers (CCBs), which was the active comparator. Drug prescriptions were ascertained using recorded British National Formulary (BNF) codes.

In the primary analysis, all three cohorts were mutually exclusive and patients having a concurrent prescription of any two of the three medications were excluded. To avoid any residual effect of any of the other two medications in their respective cohorts, those with a preceding prescription of any of the other two medications after 30th October 2019 (3 month washout period) were excluded. However, patients were still included if they had a concurrent prescription of other antihypertensive classes (e.g. diuretics and beta-blockers), but these variables were used to propensity score match the exposure and comparator cohort and further adjusted for in the outcome analysis.

\section{Matching}

We estimated propensity scores for prescription of the treatment of interest (ACE inhibitor/ARB) using logistic regression, including the covariates listed below. Matched paired exposure groups (ACE inhibitors vs CCBs and ARBs vs CCBs) were created after performing 1:1 propensity score matching using the nearest-neighbour algorithm, considering calipers of width equal to 0.2 . We matched without replacement. We assessed the covariate balance of the matched groups by calculating the standardized absolute mean difference (SMD), considering SMD below 0.10 a balanced covariate.

\section{Outcomes}

The primary outcome was a composite of confirmed or suspected diagnosis of COVID-19 recorded using the clinical (Read) codes recommended in national guidelines (clinical codes are listed in Supplementary Table 1) [17]. Confirmed SARS-CoV-2 infection was defined by a positive reverse transcriptase polymerase chain reaction (RT-PCR) swab result. Clinicians entered a suspected SARS-CoV-2 infection code when there was no RT-PCR testing available in the presence of either a compatible clinical picture, confirmation with other investigations (e.g. imaging) or contact with a confirmed case. A recent study on suspected COVID-19 codes recorded in primary care suggested that clinical diagnosis of COVID-19 by physicians followed a similar trend to test positive cases confirmed by the UK national testing service [18].

We also assessed all-cause mortality as a secondary outcome. A negative control was used to assess for residual confounding [19]. This was a composite of accidents, trauma or fractures, which was chosen on the basis that we did not expect it to be differentially associated with either the drugs of interest or the outcome.

\section{Follow-up period}

Patients were followed up from the 30th January 2020 until the earliest of the following: recording of the outcome (as defined above), death, patient left practice/ dataset, practice ceased contributing to the database, and study end (22nd July 2020). The latest available baseline covariate data recorded before 30th January 2020 were obtained. We retrospectively captured outcome records available until the study end date.

\section{Covariates}

Baseline covariates were extracted for propensity score matching and model adjustment, which included:

1) Sociodemographic characteristics - age and sex.

2) Lifestyle risk factors and metabolic profile: smoking status, alcohol consumption, body mass index (BMI), blood pressure, total cholesterol, high density lipoprotein (HDL), and estimated glomerular filtration rate (eGFR).

3) Duration of hypertension and age of hypertension diagnosis.

4) Presence of comorbid conditions including those listed as high risk for COVID-19 [12, 13]: chronic respiratory disease (including severe asthma and 
COPD), atrial fibrillation, rheumatoid arthritis, cancers (excluding skin cancer), haematological conditions (including haematological malignancies) and immunosuppressive conditions (including immunodeficiency, use of immunosuppressive drugs, chemotherapy and radiotherapy, antibody treatment for cancer, and solid organ transplant).

5) Concurrent prescriptions for thiazide diuretics, potassium diuretics, alpha-adrenoceptor blockers, beta-adrenoceptor blockers, other antihypertensives, statins, and anticoagulants, as defined based on BNF chapters.

\section{Sample size}

The study sample size was not determined by an a priori sample size calculation. Rather, we included all patients meeting the study eligibility criteria. There have already been over 300,000 confirmed COVID-19 diagnoses recorded in the UK general population. Considering the prevalence of hypertension to be $30 \%$ [20], and the THIN database constituting more than 2 million active patients, we expected to have sufficient power to detect differences in the incidence rates of the primary outcome. We included all current users of the exposure drugs as described in the section above, minimizing selection bias by using the maximum sample size available.

\section{Statistical analysis}

We used basic descriptive statistics to summarize the characteristics of the patients in each of the prescription cohorts before and after propensity score matching. Crude incidence rates of each outcome were calculated with 95\% confidence intervals (CIs). In the primary analysis, we applied a Cox proportional hazards regression model to determine crude and adjusted hazard ratios (HR) comparing pairs of treatment groups in patients with hypertension. The models were adjusted for the covariates listed above. The Cox proportional hazards assumption was tested using Schoenfeld's residuals test and log-log plots. P-values below 0.05 were considered statistically significant.

\section{Sensitivity analyses}

We repeated the primary analyses comparing outcomes for patients who used ACE inhibitors with or without CCBs to those prescribed CCBs without a RAS inhibitor. We did the same with patients prescribed ARBs with or without a CCB to those prescribed a CCB. This enabled the sample size to be increased while still assessing the additional effect of the exposure drug over and above the active comparator.

The primary analysis was also repeated including patients with diabetes mellitus, ischemic heart disease, stroke or TIA and peripheral vascular disease, mirroring the inclusion criteria used by Fosbøl et al. [16]. We also repeated the analysis further including patients with hypertension, CKD and heart failure, as was done in the study by Mancia et al. [15, 19-21]. This allowed us to assess the potential effect of confounding by indication bias introduced by including patients with different indications for the exposure drugs.

\section{Missing data}

Continuous variables such as age, BMI, and total cholesterol were grouped into clinically meaningfully categories. Missing values for smoking status and other categorical variables were treated as a separate missing categorical variable. The absence of a record of any diagnosis (e.g. hypertension, renal disease) was taken to indicate the absence of these conditions.

\section{Ethical approval}

The THIN data collection scheme and research carried out using THIN data were approved by the NHS SouthEast Multicentre Research Ethic Committee in 2003. Under the terms of the approval, studies must undergo independent scientific review. Approval for this study was obtained from the THIN Scientific Review Committee in June 2020 (SRC protocol reference 20-003-R2).

\section{Results}

\section{Population selection}

Before matching, there were 31,194 individuals with a prescription for ACE inhibitors, 13,377 with a prescription for ARBs, and 27,500 with a prescription for CCBs at the index date. After matching, there were 18,895 patients in each arm of the ACE inhibitor and $\mathrm{CCB}$ paired cohorts and 10,623 in each arm of the ARB and $C C B$ paired cohorts (Fig. 1). The median follow-up was 0.48 (interquartile range 0.48 to 0.48 ) years.

\section{Study participants ACE inhibitors versus calcium channel blockers}

Before matching, the mean age of users of ACE inhibitors and CCBs was 60.8 years and 67.4, respectively (Table 1). The proportion that were male was slightly higher for users of ACE inhibitors than users of CCBs (48.8\% vs $45.6 \%$, respectively). A similar proportion were current smokers but a greater proportion of users of ACE inhibitors were overweight or obese compared to users of CCBs (77.7\% vs $69.1 \%$, respectively).

Duration of hypertension was slightly longer for users of ACE inhibitors than CCBs (9.8 years vs 8.6 years, respectively). Systolic and diastolic blood pressure (BP), cholesterol, and renal function were similar across both groups. The prevalence of comorbidities was also similar between groups, except for cancers and respiratory 


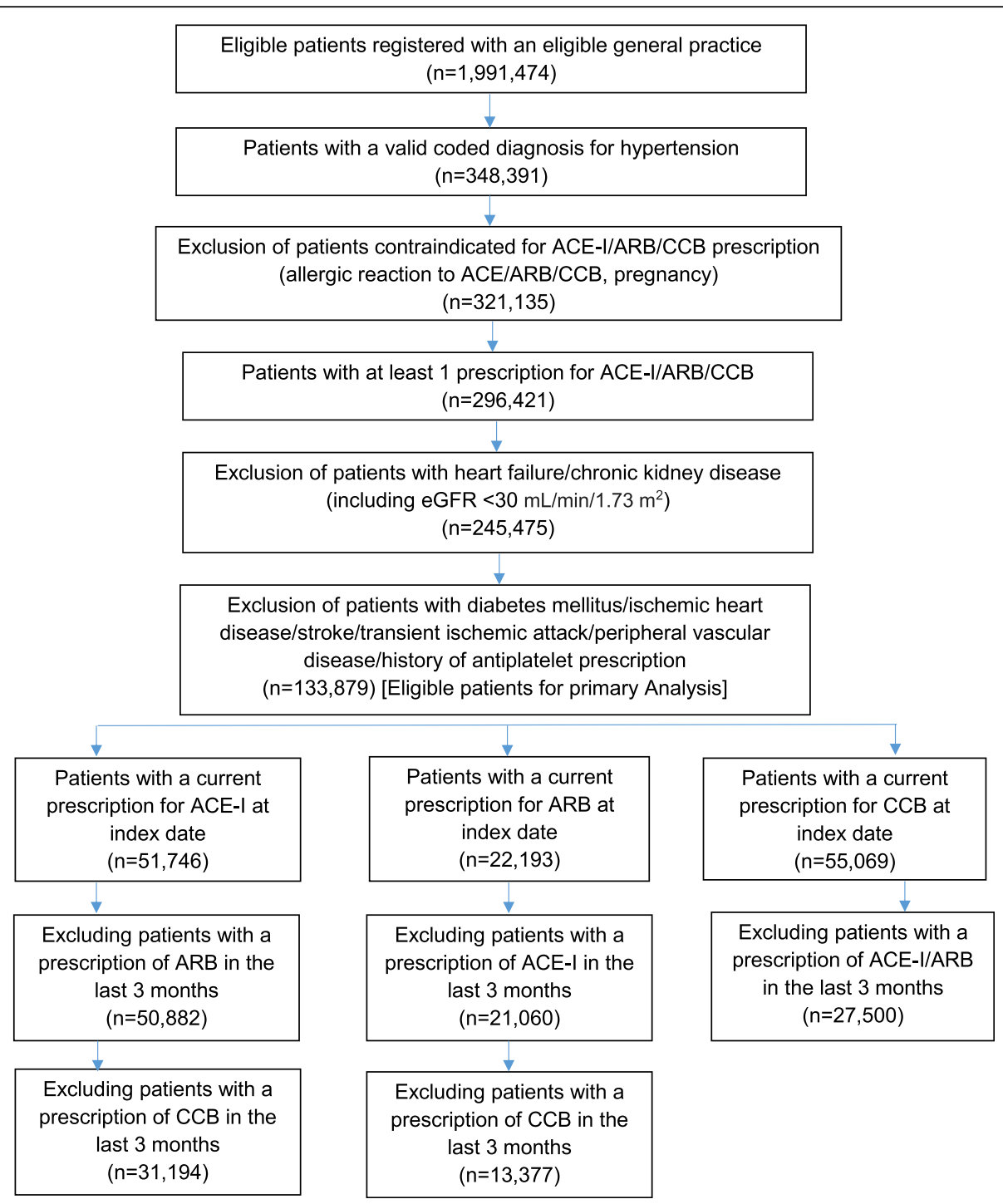

Fig. 1 Number of subjects at each stage of the study

disease, which were slightly more common in the $\mathrm{CCB}$ cohort.

$21.9 \%$ of the $\mathrm{CCB}$ cohort had previously used an ACE inhibitor and $26.4 \%$ of the ACE inhibitor cohort had previously used a CCB. Prescription of other antihypertensives was similar between groups except for thiazide diuretics, which was slightly more common in the ACE inhibitor cohort (18.5\% in users of ACE inhibitors vs $14.3 \%$ in users of CCBs). Prescriptions of statins were also similar between both cohorts.

Following propensity score matching, users of ACE inhibitors and CCBs were similar in age (64.7 years vs 63.4 years, respectively). Other characteristics were well balanced, including demographic, behavioural and metabolic risk factors, comorbidities and prescriptions (Table 1).

\section{Angiotensin II receptor blockers versus calcium channel blockers}

Before matching, the mean age of users of ARBs was younger than users of CCBs [63.8 years vs 67.4, respectively (Table 1)]. However, the proportion of males was slightly lower in users of ARBs than CCBs $(40.2 \%$ vs $45.6 \%$, respectively). A smaller proportion of users of ARBs were current smokers compared to users of CCBs (7.9\% vs $12.7 \%$, respectively). A greater proportion of users of ARBs were overweight or obese than users of CCBs $(79.1 \%$ vs $69.1 \%)$.

The ARB cohort had a longer mean duration of hypertension than the CCB cohort (11.4 years vs 8.6 , respectively) and a younger mean age at hypertension diagnosis (52.4 years vs 58.8). BP, cholesterol and renal function were similar between groups, as was the prevalence of comorbidities. 
Table 1 Baseline characteristics of the primary analysis cohorts

\begin{tabular}{|c|c|c|c|c|c|c|c|c|}
\hline \multirow[t]{2}{*}{ Primary Analysis Cohort } & \multicolumn{2}{|l|}{ Unmatched } & \multicolumn{2}{|c|}{$\begin{array}{l}\text { Propensity score } \\
\text { matched }\end{array}$} & \multicolumn{2}{|l|}{ Unmatched } & \multicolumn{2}{|c|}{$\begin{array}{l}\text { Propensity score } \\
\text { matched }\end{array}$} \\
\hline & $\begin{array}{l}\text { ACE-I } \\
(n=31,194)\end{array}$ & $\begin{array}{l}\text { CCB } \\
(n=27,500)\end{array}$ & $\begin{array}{l}\text { ACE-I } \\
(n=18,895)\end{array}$ & $\begin{array}{l}\text { CCB } \\
(n=18,895)\end{array}$ & $\begin{array}{l}\text { ARB } \\
(n=13,377)\end{array}$ & $\begin{array}{l}\text { CCB } \\
(n=27,500)\end{array}$ & $\begin{array}{l}\text { ARB } \\
(n=10,623)\end{array}$ & $\begin{array}{l}\text { CCB } \\
(n=10,623)\end{array}$ \\
\hline Age, mean (SD) & $60.8(11.7)$ & $67.4(10.8)$ & $64.7(11.2)$ & $63.4(9.6)$ & $63.8(11.3)$ & $67.4(10.8)$ & $65.0(11.1)$ & $64.55(11.5)$ \\
\hline \multicolumn{9}{|l|}{ Age categories, n (\%) } \\
\hline 18-30 years & $123(0.4)$ & $28(0.1)$ & $34(0.2)$ & $28(0.2)$ & $23(0.2)$ & $28(0.1)$ & $14(0.1)$ & $26(0.2)$ \\
\hline $30-40$ years & $875(2.8)$ & $309(1.1)$ & $213(1.1)$ & $308(1.6)$ & $206(1.5)$ & $309(1.1)$ & $118(1.1)$ & $238(2.2)$ \\
\hline $40-50$ years & $4466(14.3)$ & $1302(4.7)$ & $1445(7.7)$ & $1297(6.9)$ & $1298(9.7)$ & $1302(4.7)$ & $834(7.9)$ & $865(8.1)$ \\
\hline $50-60$ years & $10,214(32.7)$ & $4836(17.6)$ & $5018(26.6)$ & $4694(24.8)$ & 3556 (26.6) & 4836 (17.6) & $2623(24.7)$ & $2472(23.3)$ \\
\hline $60-70$ years & $8448(27.1)$ & $9521(34.6)$ & $5879(31.1)$ & 7969 (42.2) & 4157 (31.1) & $9521(34.6)$ & 3388 (31.9) & 3497 (32.9) \\
\hline $70-80$ years & $5380(17.3)$ & $8376(30.5)$ & $4666(24.7)$ & $3974(21.0)$ & 3097 (23.2) & $8376(30.5)$ & $2703(25.4)$ & $2617(24.6)$ \\
\hline$>80$ years & $1688(5.4)$ & $3128(11.4)$ & $1640(8.7)$ & $625(3.3)$ & $1040(7.8)$ & $3128(11.4)$ & $943(8.9)$ & $908(8.6)$ \\
\hline Sex (Male), n (\%) & $15,213(48.8)$ & $12,547(45.6)$ & $8870(46.9)$ & $9243(48.9)$ & $5379(40.2)$ & $12,547(45.6)$ & $4628(43.6)$ & $4463(42.0)$ \\
\hline \multicolumn{9}{|l|}{ Smoker categories, n (\%) } \\
\hline Non Smoker & $17,968(57.6)$ & $15,514(56.4)$ & $10,725(56.8)$ & $10,668(56.5)$ & $8390(62.7)$ & $15,514(56.4)$ & $6384(60.1)$ & $6473(60.9)$ \\
\hline Ex-Smoker & $9073(29.1)$ & 8329 (30.3) & $5677(30.0)$ & $5700(30.2)$ & 3865 (28.9) & $8329(30.3)$ & $3186(30.0)$ & 3137 (29.5) \\
\hline Smoker & $3962(12.7)$ & $3483(12.7)$ & $2402(12.7)$ & 2431 (12.9) & $1058(7.9)$ & $3483(12.7)$ & $1005(9.5)$ & $967(9.1)$ \\
\hline Missing & $191(0.6)$ & $174(0.6)$ & $91(0.5)$ & $96(0.5)$ & $64(0.5)$ & $174(0.6)$ & $48(0.5)$ & $46(0.4)$ \\
\hline \multicolumn{9}{|l|}{ Drinker Categories, n (\%) } \\
\hline Non-drinker & $4716(15.1)$ & $4186(15.2)$ & $2874(15.2)$ & $2856(15.1)$ & $2013(15.1)$ & $4186(15.2)$ & $1587(14.9)$ & $1508(14.2)$ \\
\hline Drinker without excess & $11,716(37.6)$ & $10,281(37.4)$ & $7082(37.5)$ & $7134(37.8)$ & 5157 (38.6) & $10,281(37.4)$ & $4023(37.9)$ & 3976 (37.4) \\
\hline Excessive Drinker & $7614(24.4)$ & $6238(22.7)$ & 4464 (23.6) & $4673(24.7)$ & $2908(21.7)$ & $6238(22.7)$ & $2414(22.7)$ & $2368(22.3)$ \\
\hline Missing & 7148 (22.9) & $6795(24.7)$ & $4475(23.7)$ & $4232(22.4)$ & $3299(24.7)$ & $6795(24.7)$ & $2599(24.5)$ & $2771(26.1)$ \\
\hline BMI, mean (SD) & $30.3(6.4)$ & $28.44(5.6)$ & $29.36(6.0)$ & $29.48(5.7)$ & $30.37(6.2)$ & $28.44(5.6)$ & $29.58(5.9)$ & $29.65(5.9)$ \\
\hline \multicolumn{9}{|l|}{ BMI Categories, n (\%) } \\
\hline Underweight $(<18.5)$ & $173(0.6)$ & $343(1.3)$ & $151(0.8)$ & $105(0.6)$ & $67(0.5)$ & $343(1.3)$ & $65(0.6)$ & $62(0.6)$ \\
\hline Underweight $(<25)$ & $5597(17.9)$ & $6898(25.1)$ & 4059 (21.5) & 3645 (19.3) & $2243(16.8)$ & $6898(25.1)$ & $2074(19.5)$ & $2033(19.1)$ \\
\hline Overweight (25-30) & $10,948(35.1)$ & $10,536(38.3)$ & 7156 (37.9) & $7171(38.0)$ & 4718 (35.3) & $10,536(38.3)$ & 4055 (38.2) & 3894 (36.7) \\
\hline Obese (> 30) & $13,300(42.6)$ & $8470(30.8)$ & $6771(35.8)$ & 7271 (38.5) & $5854(43.8)$ & 8470 (30.8) & 4004 (37.7) & 4185 (39.4) \\
\hline Missing & $1176(3.8)$ & $1253(4.6)$ & $758(4.0)$ & $703(3.7)$ & 495 (3.7) & $1253(4.6)$ & $425(4.0)$ & 449 (4.2) \\
\hline $\begin{array}{l}\text { Hypertension duration, mean years } \\
\text { (SD) }\end{array}$ & $9.8(7.4)$ & $8.6(7.5)$ & $9.4(6.9)$ & $9.4(8.0)$ & $11.4(7.5)$ & $8.6(7.5)$ & $10.5(7.0)$ & $11.3(8.3)$ \\
\hline $\begin{array}{l}\text { Age at hypertension diagnosis, } \\
\text { mean (SD) }\end{array}$ & $51.0(10.9)$ & $58.8(11.2)$ & $55.3(10.4)$ & $54.0(9.1)$ & $52.4(10.9)$ & $58.8(11.2)$ & $54.5(10.4)$ & $53.2(11.0)$ \\
\hline Systolic BP, mean (SD) & $135.9(13.8)$ & $137.3(13.6)$ & $136.7(14.0)$ & $136.4(13.1)$ & $136.3(13.6)$ & $137.3(13.6)$ & $136.8(13.6)$ & $136.6(13.4)$ \\
\hline Diastolic BP, mean (SD) & $81.7(9.4)$ & $79.76(9.3)$ & $80.6(9.3)$ & $81.09(9.0)$ & $80.8(9.1)$ & $79.8(9.3)$ & $80.5(9.2)$ & $80.5(9.3)$ \\
\hline Missing, n (\%) & $80(0.3)$ & $63(0.2)$ & $0(0 \%)$ & $0(0 \%)$ & $22(0.2)$ & $63(0.2)$ & $0(0 \%)$ & $0(0 \%)$ \\
\hline Cholesterol, mean (SD) & $5.13(1.03)$ & $5.13(1.04)$ & $5.12(1.04)$ & $5.13(1.04)$ & $5.13(1.01)$ & $5.13(1.04)$ & $5.14(1.02)$ & $5.13(1.03)$ \\
\hline \multicolumn{9}{|l|}{ Cholesterol Categories, n (\%) } \\
\hline$<5.2 \mathrm{mmol} / \mathrm{L}$ & $17,051(54.7)$ & $14,915(54.2)$ & $10,430(55.2)$ & $10,394(55.0)$ & $7415(55.4)$ & $14,915(54.2)$ & $5820(54.8)$ & $5894(55.5)$ \\
\hline $5.2-6.2 \mathrm{mmol} / \mathrm{L}$ & $8852(28.4)$ & $7712(28.0)$ & $5262(27.9)$ & $5323(28.2)$ & $3836(28.7)$ & $7712(28.0)$ & $3026(28.5)$ & 2929 (27.6) \\
\hline$>=6.2 \mathrm{mmol} / \mathrm{L}$ & $4153(13.3)$ & 3747 (13.6) & $2543(13.5)$ & $2580(13.7)$ & $1699(12.7)$ & 3747 (13.6) & 1419 (13.4) & $1404(13.2)$ \\
\hline Missing & $1138(3.7)$ & $1126(4.1)$ & $660(3.5)$ & $598(3.2)$ & $427(3.2)$ & $1126(4.1)$ & $358(3.4)$ & $396(3.7)$ \\
\hline $\mathrm{HDL}$, mean (SD) & $1.44(0.43)$ & $1.54(0.46)$ & $1.49(0.44)$ & $1.48(0.44)$ & $1.48(0.43)$ & $1.54(0.46)$ & $1.50(0.44)$ & $1.50(0.45)$ \\
\hline
\end{tabular}


Table 1 Baseline characteristics of the primary analysis cohorts (Continued)

\begin{tabular}{|c|c|c|c|c|c|c|c|c|}
\hline \multirow[t]{2}{*}{ Primary Analysis Cohort } & \multicolumn{2}{|l|}{ Unmatched } & \multicolumn{2}{|c|}{$\begin{array}{l}\text { Propensity score } \\
\text { matched }\end{array}$} & \multicolumn{2}{|l|}{ Unmatched } & \multicolumn{2}{|c|}{$\begin{array}{l}\text { Propensity score } \\
\text { matched }\end{array}$} \\
\hline & $\begin{array}{l}\text { ACE-I } \\
(n=31,194)\end{array}$ & $\begin{array}{l}\text { CCB } \\
(n=27,500)\end{array}$ & $\begin{array}{l}\text { ACE-I } \\
(n=18,895)\end{array}$ & $\begin{array}{l}\text { CCB } \\
(n=18,895)\end{array}$ & $\begin{array}{l}\text { ARB } \\
(n=13,377)\end{array}$ & $\begin{array}{l}\text { CCB } \\
(n=27,500)\end{array}$ & $\begin{array}{l}\text { ARB } \\
(n=10,623)\end{array}$ & $\begin{array}{l}\text { CCB } \\
(n=10,623)\end{array}$ \\
\hline \multicolumn{9}{|l|}{ HDL categories, n (\%) } \\
\hline$<1.55 \mathrm{mmol} / \mathrm{L}$ & $19,720(63.2)$ & $14,873(54.1)$ & $10,949(58.0)$ & $\begin{array}{l}11,464(60 . \\
7)\end{array}$ & $8160(61.0)$ & $14,873(54.1)$ & $6214(58.5)$ & $6251(58.8)$ \\
\hline$>=1.55 \mathrm{mmol} / \mathrm{L}$ & 9851 (31.6) & $11,114(40.4)$ & 7007 (37.1) & $6566(34.8)$ & $4621(34.5)$ & $11,114(40.4)$ & $3915(36.9)$ & $3818(35.9)$ \\
\hline Missing & $1623(5.20)$ & $1513(5.5)$ & $939(5.0)$ & $865(4.6)$ & $596(4.5)$ & $1513(5.5)$ & $494(4.7)$ & $554(5.2)$ \\
\hline eGFR, mean (SD) & $86.1(14.7)$ & $82.8(13.8)$ & $83.33(14.3)$ & $85.08(14.0)$ & $82.9(14.6)$ & $82.8(13.8)$ & $82.36(14.2)$ & $83.89(14.7)$ \\
\hline \multicolumn{9}{|l|}{ eGFR category, n (\%) } \\
\hline$>60$ (Stage 2 and above) & $29,735(95.3)$ & $25,680(93.4)$ & $17,806(94.2)$ & $17,924(94.9)$ & $12,538(93.7)$ & $25,680(93.4)$ & $9981(94.0)$ & 9927 (93.5) \\
\hline 30-59(Stage 3) & $1127(3.6)$ & $1270(4.6)$ & $847(4.5)$ & $792(4.2)$ & $713(5.3)$ & $1270(4.6)$ & $530(5.0)$ & $578(5.4)$ \\
\hline Missing & $332(1.1)$ & $550(2.0)$ & $242(1.3)$ & $179(1.0)$ & $126(0.9)$ & $550(2.0)$ & $112(1.1)$ & $118(1.1)$ \\
\hline \multicolumn{9}{|l|}{ Baseline conditions, n (\%) } \\
\hline Atrial fibrillation & $726(2.3)$ & $743(2.7)$ & $503(2.7)$ & $502(2.7)$ & $384(2.9)$ & $743(2.7)$ & $318(3.0)$ & $332(3.1)$ \\
\hline Rheumatoid arthritis & $460(1.5)$ & $497(1.8)$ & $329(1.7)$ & 297 (1.6) & $199(1.5)$ & $497(1.8)$ & $161(1.5)$ & $166(1.6)$ \\
\hline Cancer & $2525(8.1)$ & $3320(12.1)$ & $1921(10.2)$ & $1711(9.1)$ & $1377(10.3)$ & $3320(12.1)$ & $1151(10.8)$ & $1139(10.7)$ \\
\hline Respiratory Disease & $1325(4.3)$ & $1695(6.2)$ & $1010(5.4)$ & $933(4.9)$ & $664(5.0)$ & $1695(6.2)$ & $586(5.5)$ & $572(5.4)$ \\
\hline Immunosuppressed ${ }^{a}$ & $571(1.8)$ & $616(2.2)$ & $392(2.1)$ & $358(1.9)$ & $239(1.8)$ & $616(2.2)$ & $205(1.9)$ & $198(1.9)$ \\
\hline Rare metabolic disorder & $29(0.1)$ & $38(0.1)$ & $23(0.1)$ & $18(0.1)$ & $18(0.1)$ & $38(0.1)$ & $15(0.1)$ & $13(0.1)$ \\
\hline \multicolumn{9}{|l|}{ Other medications at baseline, n (\%) } \\
\hline Previous prescription of ACE-I & $31,194(100)$ & $6030(21.9)$ & $18,895(100)$ & $4462(23.6)$ & $8421(63.0)$ & $6030(21.9)$ & $5686(53.5)$ & $5638(53.1)$ \\
\hline Previous prescription of ARB & $1036(3.3)$ & $1907(6.9)$ & $937(5.0)$ & $762(4.0)$ & $13,377(100)$ & $1907(6.9)$ & $10,623(100)$ & $1492(14.0)$ \\
\hline Previous prescription of CCB & $8242(26.4)$ & $27,500(100)$ & $5554(29.4)$ & $18,895(100)$ & $5393(40.3)$ & $27,500(100)$ & $4001(37.7)$ & $10,623(100)$ \\
\hline Thiazide diuretics & $5775(18.5)$ & 3919 (14.3) & $5775(18.5)$ & 3919 (14.3) & 3457 (25.8) & 3919 (14.3) & $2074(19.5)$ & $2468(23.2)$ \\
\hline Loop diuretics & $768(2.5)$ & $461(1.7)$ & $768(2.5)$ & $461(1.7)$ & $417(3.1)$ & $461(1.7)$ & $331(3.1)$ & $219(2.1)$ \\
\hline Potassium diuretics & $148(0.5)$ & $101(0.4)$ & $148(0.5)$ & $101(0.4)$ & $85(0.6)$ & $101(0.4)$ & $59(0.6)$ & $69(0.7)$ \\
\hline Alpha blockers & $1017(3.3)$ & $659(2.4)$ & $1017(3.3)$ & $659(2.4)$ & $661(4.9)$ & $659(2.4)$ & $430(4.1)$ & $481(4.5)$ \\
\hline Beta blockers & 3525 (11.3) & $3030(11.0)$ & 3525 (11.3) & $3030(11.0)$ & $1630(12.2)$ & $3030(11.0)$ & $1302(12.3)$ & $1423(13.4)$ \\
\hline Other antihypertensive & $122(0.4)$ & $84(0.3)$ & $122(0.4)$ & $84(0.3)$ & $71(0.5)$ & $84(0.3)$ & $48(0.5)$ & $48(0.5)$ \\
\hline Anticoagulants & $884(2.8)$ & $889(3.2)$ & $884(2.8)$ & $889(3.2)$ & $446(3.3)$ & $889(3.2)$ & $370(3.5)$ & 385 (3.6) \\
\hline Statins & 9399 (30.1) & $9133(33.2)$ & $9399(30.1)$ & $9133(33.2)$ & 3977 (29.7) & 9133 (33.2) & $3293(31.0)$ & 3230 (30.4) \\
\hline
\end{tabular}

a (Treatment with immunosuppressive therapies/antibody treatment/solid organ transplant/ chemo/radiotherapies)

$A C E-I$ angiotensin converting enzyme inhibitor, $A R B$ angiotensin II receptor blocker, $C C B$ calcium channel blocker

63.0\% of users of ARBs had previously used an ACE inhibitor, compared to $21.9 \%$ of users of CCBs. $40.3 \%$ of the ARB cohort had previously used a CCB while $6.9 \%$ of the CCB cohort had previously used an ARB. Users of ARBs were more likely than users of CCBs to have been prescribed thiazide diuretics as well as other antihypertensive drugs. However, the proportion with a prescription for statins was slightly greater in the CCB cohort.

Following propensity score matching, characteristics were well balanced between both groups, including demographic characteristics, behavioural risk factors, metabolic profile, comorbidities, and prescriptions (Table 1).

\section{Outcomes}

\section{ACE inhibitors versus calcium channel blockers}

Before matching, 148 individuals (0.47\%) in the ACE inhibitor cohort developed suspected or confirmed COVID-19 during 14,733 person-years of follow-up, representing a crude incidence rate of 10.1 per 1000 person-years (Table 2). One hundred twenty-six individuals $(0.46 \%)$ in the CCB cohort developed suspected or confirmed COVID-19 during 12,985 person-years, representing a crude incidence rate of 9.70 per 1000 person-years in the CCB cohort. The unadjusted hazard ratio for suspected/ confirmed COVID-19 comparing the ACE inhibitor cohort to the CCB cohort (as the reference) was 1.04 (95\% CI 0.82 


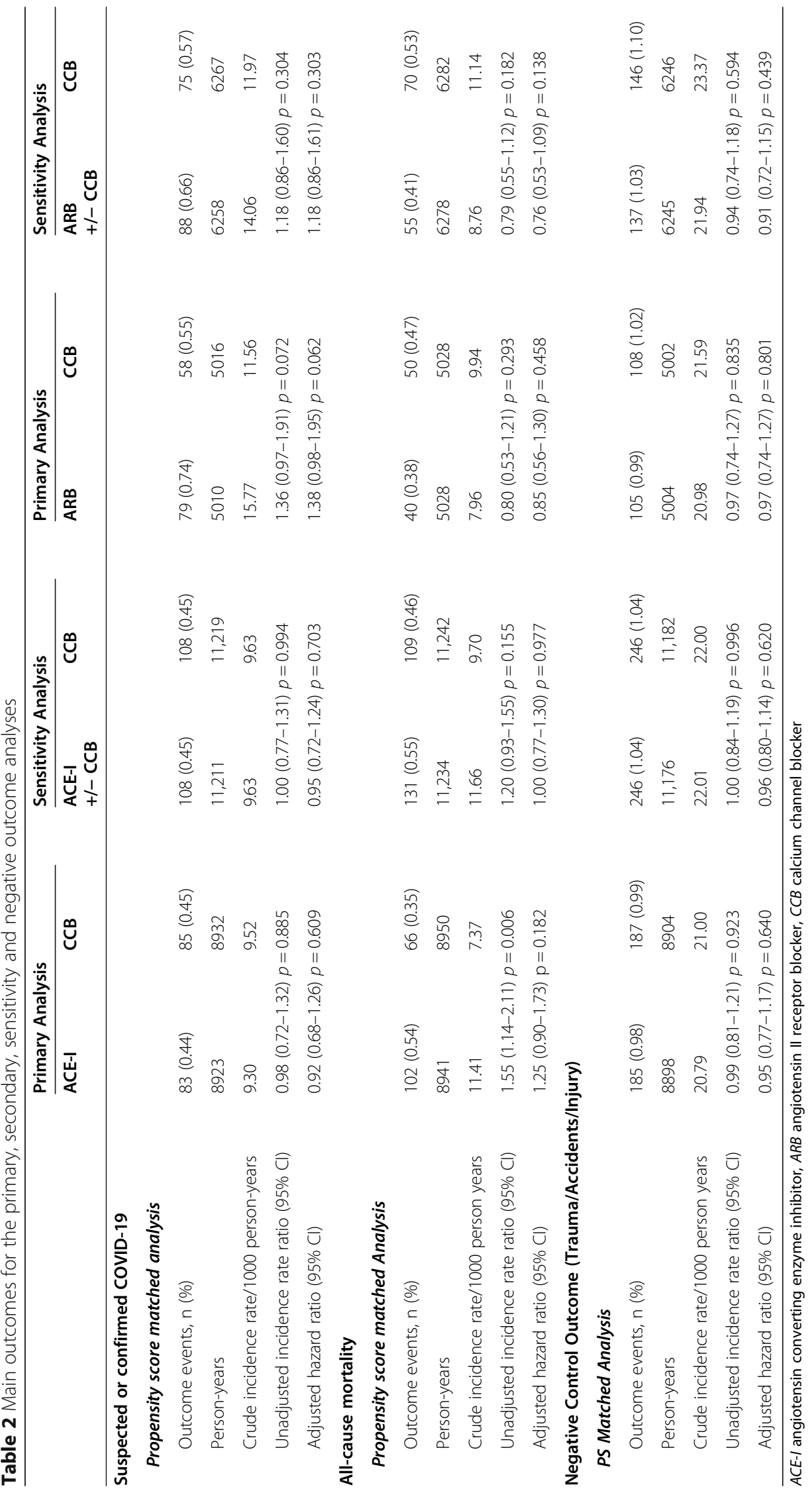


aHR $[95 \% \mathrm{Cl}]$

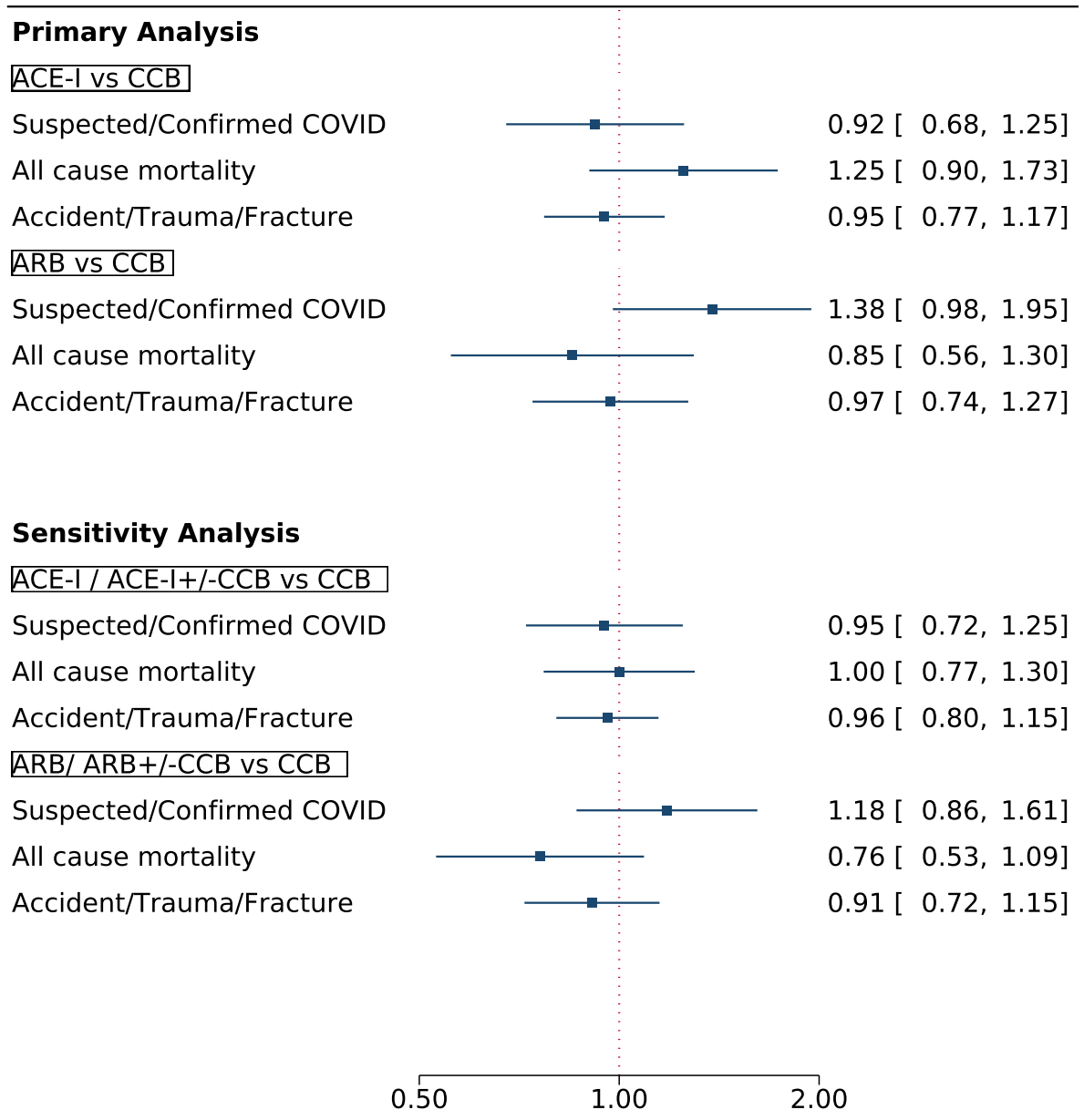

$A C E-I=a n g i o t e n s i n$ converting enzyme inhibitor, $A R B=$ angiotensin II receptor blocker, $C C B=$ calcium channel blocker

Fig. 2 Forest plot of adjusted hazard ratios for suspected or confirmed COVD-19, all-cause mortality and accidents, trauma and fractures (negative control)

to 1.31 ), which fell slightly to 1.01 (95\% 0.78 to 1.30$)$ after adjusting for measured confounders.

Following propensity score matching, 83 individuals (0.44\%) in the ACE inhibitor cohort had suspected or diagnosed COVID-19 during 8923 person-years of follow-up, representing a crude incidence rate of 9.30 per 1000 person-years. Eighty five individuals (0.45\%) in the $\mathrm{CCB}$ cohort developed suspected or confirmed COVID-19 during 8932 person-years of follow-up representing a crude incidence rate of 9.5 per 1000 person-years. The unadjusted hazard ratio for suspected/confirmed COVID-19 comparing the ACE inhibitor cohort to the CCB cohort was 0.98 (95\% CI 0.72 to 1.32). Upon adjustment for measured confounders, the hazard ratio was 0.92 (95\% 0.68 to 1.26 ; Fig. 2).

Similar results were found in the sensitivity analyses for the primary outcome. When comparing hypertensive users of ACE inhibitors with or without
CCBs to those using CCBs alone, the adjusted hazard ratio was 0.95 (95\% CI 0.72 to 1.25 ) following propensity score matching. When including individuals with diabetes mellitus, ischemic heart disease, stroke, transient ischaemic attack and peripheral vascular disease, the adjusted hazard ratio for COVID19 after propensity score matching was 0.91 (95\% CI 0.74 to 1.12 ). When including individuals with any comorbidities, the adjusted hazard ratio after propensity score matching was similarly 0.98 (95\% CI 0.81 to 1.18 ; Fig. 3 ).

The propensity score-matched analysis for all-cause mortality produced a statistically non-significant adjusted hazard ratio of 1.25 (95\% CI 0.90 to 1.73 ). The negative control analysis similarly found no statistically significant association between prescription of ACE inhibitors and accidents, trauma or fractures compared to prescription of CCBs (adjusted HR 0.95, 95\% CI 0.77 to 1.17 ). 


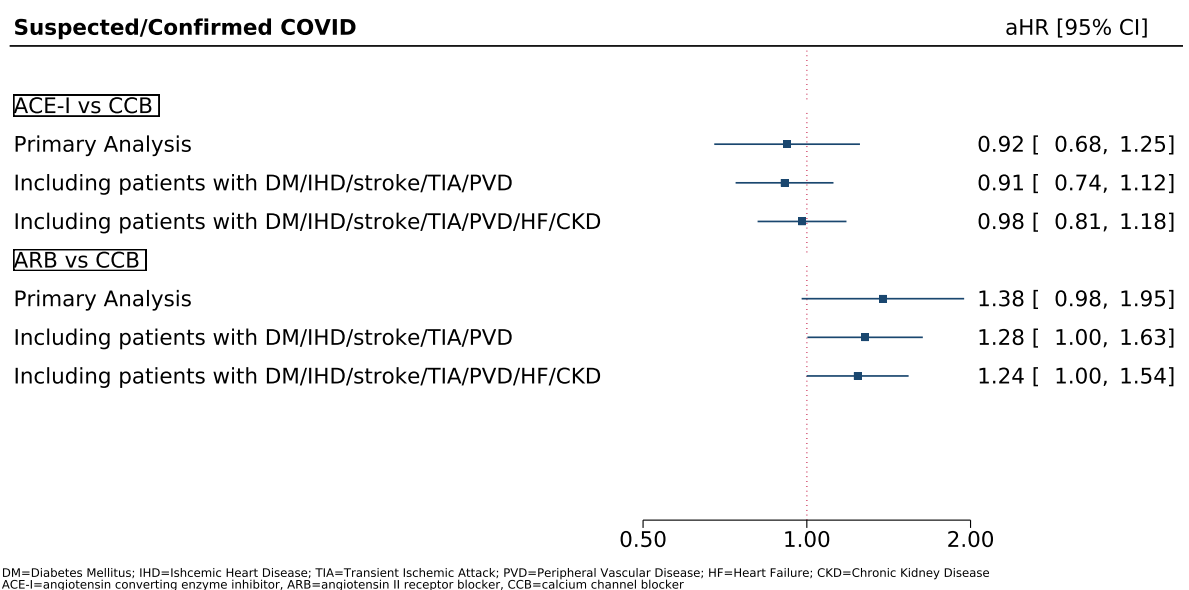

Fig. 3 Forest plot of hazard ratios for suspected or confirmed COVID-19 when including comorbidities that were excluded in the primary analysis

\section{Angiotensin II receptor blockers versus calcium channel blockers}

Before matching, 99 individuals (0.74\%) in the ARB cohort developed suspected or confirmed COVID-19 over a follow-up of 6308 person-years, representing a crude incidence rate of 15.7 per 1000 person-years. 126 $(0.46 \%)$ in the CCB cohort developed suspected or confirmed COVID-19 over a follow-up of 12,985 personyears, representing a crude incidence rate of 9.7 per 1000 person-years. The unadjusted hazard ratio for suspected or confirmed COVID-19 when comparing the ARB cohort to the CCB cohort (as the reference) was 1.62 (95\% CI 1.24 to 2.10). After adjustment for measured confounders, the hazard ratio was slightly attenuated to 1.51 (95\% CI 1.12 to 2.03), although it remained statistically significant.

After propensity score matching, 79 individuals $(0.74 \%)$ in the ARB cohort developed suspected or confirmed COVID-19 over 5010 person-years of follow-up, representing a crude incidence rate of 15.8 per 1000 person-years. In the CCB cohort, 58 individuals (0.55\%) developed suspected or confirmed COVID-19 over 5016 years of follow-up, representing a crude incidence rate of 11.6 per 1000 person-years. The unadjusted hazard ratio when comparing the ARB cohort to the CCB cohort was 1.36 (95\% CI 0.97 to 1.91). After adjustment for measured confounders, the hazard ratio increased slightly to 1.38 (95\% CI 0.98 to 1.95 ; Fig. 2).

These findings were attenuated in sensitivity analyses when comparing users of ARBs with or without concurrent use of CCBs to propensity score-matched individuals using CCBs alone (adjusted HR 1.18, 95\% CI 0.86 to 1.61). When only excluding individuals with either heart failure or CKD, the adjusted hazard ratio was slightly attenuated compared to the primary analysis but statistically significant after propensity score matching (adjusted HR 1.28, 95\% CI 1.00 to 1.63). This was similarly found when including propensity scorematched individuals with any comorbidity (adjusted HR $1.24,95 \%$ CI 1.00 to 1.54 ; Fig. 3).

There was no statistically significant difference in allcause mortality between users of ARBs compared to users of CCBs after propensity score matching (adjusted HR 0.85 , 95\% CI 0.56 to 1.30 ). Similarly, there was no association between use of ARBs and the negative control outcome of accidents, trauma and fractures when compared to propensity score matched users of CCBs (adjusted HR 0.97, 95\% CI 0.74 to 1.27 ).

\section{Discussion}

\section{Main findings}

We found no difference in the risk of developing suspected or confirmed COVID-19 or all-cause mortality among individuals with hypertension treated with ACE inhibitors compared to those treated with CCBs, after matching and adjusting for a wide range of risk factors known to be associated with COVID-19, as well as indications for ACE inhibitor prescription. We found a $38 \%$ relative increase in the risk of the development of suspected or confirmed COVID-19 among those prescribed ARBs compared to those prescribed CCBs that did not reach statistical significance in the propensity score matched analysis. We also found no difference in allcause mortality.

\section{Relationship to other studies}

There has been ongoing debate over whether RAS inhibitors are protective or harmful in the context of COVID19. Concerns were raised early on in the pandemic speculating that this class of drugs could increase susceptibility to COVID-19 by upregulating ACE2 receptors, and thus promoting entry of SARS-CoV-2 virus into host cells [21]. Two in vivo studies in rats showed that ACE inhibitors increased ACE2 activity in the 
plasma and renal cortex [22, 23]. However, these studies involved far higher doses of ACE inhibitors than would typically be used in humans. In fact, a review of 11 human studies overwhelmingly showed that RAS inhibitors do not increase plasma or urine ACE2 expression, although it remains unknown whether there is any effect on membrane-bound ACE2 activity; indeed, there are no studies to date on the effects of RAS inhibitors specifically on lung ACE2 expression [10, 24].

Once within the cell, coronaviruses themselves downregulate ACE2 expression in host cells, which is understood to reduce the pulmonary activity of the antiinflammatory ACE2/angiotensin 1-7/mas receptor system $[7,21]$. This results in angiotensin II proliferation and consequent lung inflammation. In one small study of hospitalised patients with COVID-19, angiotensin II levels were markedly elevated and linearly associated with viral load and severity of lung injury [25]. Both ACE inhibitors and ARBs have been shown to attenuate the inflammatory response in mouse models, potentially through the inhibition of interleukin 6 (IL-6) [26].

It is possible that RAS inhibitors confer a protective effect through their anti-inflammatory actions, although further evidence of such a mechanism is needed. Two small case series in humans both found that SARS-CoV2 infected patients on RAS inhibitors had significantly lower inflammatory markers, and increased CD3 and CD8 $\mathrm{T}$ cell proliferation than patients on alternative antihypertensive medications $[7,27,28]$.

More recently, meta-analyses of observational studies have also shown that RAS inhibitors are not associated with severe outcomes and death in hospitalised patients with COVID-19. In a pooled analysis of 16 studies, RAS inhibitor use was non-significantly associated with lower odds of developing severe disease (OR 0.81, 95\% CI 0.41 to 1.58 ) and mortality (OR $0.86,95 \%$ CI 0.53 to 1.41 ) [29]. Similarly, in a pooled analysis of 11 studies, Pranata et al. found a non-significantly lower adjusted odds of mortality in those on RAS inhibitors (OR 0.83, 95\% CI 0.54 to 1.27 ) but no difference for disease severity (OR $1.03,95 \%$ CI 0.73 to 1.45 ) [30]. In contrast to our findings, a subgroup analysis found that ARBs were associated with reduced mortality (OR $0.51,95 \%$ CI 0.29 to $0.90)$ but the association was not statistically significant for ACE inhibitors (OR 0.68, 95\% CI 0.39 to 1.17).

A meta-analysis of five observational studies also showed a reduced risk of critical or fatal outcomes among patients with COVID-19 who took RAS inhibitors, with a pooled odds ratio of 0.32 (95\% CI 0.22 to $0.46)$ [31]. Ghosal et al. similarly showed a reduction in the odds of severe disease and death (OR 0.56 for severe illness, $95 \%$ CI 0.34 to 1.89 , and OR 0.38 for death, $95 \%$ CI 0.19 to 0.74 ) [32]. A systematic review of RAS inhibitors and COVID-19 by Zhang et al. also showed that their use was not associated with increased likelihood of testing positive for SARS-CoV-2 or with severity of disease once infected [33].

The studies included in these meta-analyses were largely based on hospital cohorts, in contrast to our study which included patients registered in primary care. Prescriptions of and compliance with prehospital medications may be much better recorded in primary care cohorts. Hospital-based cohorts typically include the most severely ill patients and do not include those with asymptomatic or mild-to-moderate disease. Furthermore, many of the included studies did not adjust effect estimates for potential confounding factors or assess the effects of sub-classes of RAS inhibitors (ACE inhibitors and ARBs) separately, which our study suggests may not be uniform.

Mancia et al. conducted a large population-based study in Lombardy of those diagnosed with COVID-19 matched to population controls on age, sex and geography [15]. After multivariable adjustment, neither ACE inhibitors nor ARBs showed an association with the risk of developing COVID-19 (OR 0.95 [95\% CI 0.86 to 1.05] and 0.96 [95\% CI 0.87 to 1.07], respectively. Another large retrospective cohort and nested case-control study of all Danish people assessed in hospital with COVID-19 in a 3 month period found no association between ACE inhibitor/ARB use and susceptibility to COVID-19 or mortality when compared with other antihypertensives [16].

A large multinational cohort study, which used propensity score matching and negative controls found, as we did, that prescription of ACE inhibitors or ARBs was not associated with the risk of diagnosis of COVID-19 in comparison to use of calcium channel blockers or thiazide diuretics [34]. When directly comparing invididuals prescribed ACE inhibitors with those prescribed ARBs, there was a higher risk of COVID-19 diagnosis in the latter. However, there were no significant differences in COVID-19 related hospitalisation between all antihypertensive drug classes.

Most recently, a prospective cohort study using data from general practices in England found that use of ACE inhibitors or ARBs was associated with a significantly reduced risk of COVID-19 but not associated ICU admission [35]. This study also found that ethnicity modified the association between use of RAS inhibitors and COVID-19, with those from Black ethnic groups being at increased risk, a trend we were not able to explore in our study.

The above studies included patients with a range of comorbidities that were potential indications for RAS inhibitors and were therefore potentially prone to prescription by indication bias. We limited our inclusion criteria to patients with hypertension and excluded 
those with other conditions that were potential indications for RAS inhibitors in our main analysis to limit the effect of these biases. However, our findings remained largely in line with these prior studies.

\section{Strengths and limitations}

The primary outcome of suspected or confirmed COVID-19 may not have been well recorded in primary care records. The current cumulative incidence of COVID-19 in the UK is 5.6\%, whereas the incidence in our cohorts ranged from 0.44 to $0.74 \%$. Relatively little testing for COVID-19 occurred early in the pandemic and data flows from COVID-19 testing centres and hospitals to primary care has generally been suboptimal. However, we expect this effect to have been equally distributed across all our included drug exposure cohorts and it should therefore not have biased our effect estimates. Furthermore, the higher cumulative incidence of COVID-19 currently reported in the UK also includes asymptomatic cases derived from increased community testing that had not been occurring earlier on in the pandemic in the period when our cohorts were derived.

We did not have access to data on hospitalisations or cause-specific mortality. Because of the low numbers of deaths in each drug exposure cohort, we did not have sufficient statistical power to assess the association between drug exposures and COVID-19 mortality. We also had insufficient data on ethnicity and socioeconomic status to include this in our analyses, both of which are known to be associated with COVID-19.

The strengths of the study include the study design, which attempted to control for confounding by indication bias and adjust for a large number of known risk factors for COVID-19. We also performed multiple sensitivity analyses to check the robustness of our findings in comparison with other published studies.

\section{Implications for practice, policy and research}

Despite initial concerns about the safety of ACE inhibitors in the context of SARS-CoV-2 pathophysiology, they appear to have no effect on susceptibility to COVID-19 compared to the use of CCBs. Our findings should provide further reassurance, in addition to previously published studies on this topic, that prescription of ACE inhibitors does not increase vulnerability to being infected with SARS-CoV-2.

However, our findings suggest that the effects of RAS inhibitors are not uniform across drug classes. ARBs by contrast were associated with a statistically non-significant increased risk of presentation with COVID-19, but not mortality, in comparison to the use of CCBs. The reasons for this are unclear but one potential reason may be due to differential health-seeking behaviour between users of ACE inhibitors and ARBs. One common side effect of ACE inhibitors is cough due to the reduced breakdown of bradykinin and substance $\mathrm{P}$, which are degraded by $\mathrm{ACE}$, and a rise in prostaglandins due to increased concentrations of bradykinin [36]. Patients who experience cough secondary to ACE inhibitors are frequently switched to ARBs, and indeed a large proportion of patients in our ARB cohort had been previously prescribed an ACE inhibitor. Those prescribed ARBs could therefore be more prone to coughing, which could, in turn, have made them more likely to present to healthcare services with symptoms of COVID-19. Reassuringly, we did not see any association between use of ARBs and all-cause mortality during the peak of the pandemic, supporting the hypothesis that the association between their use and COVID-19 is likely related to differences in health seeking behaviour rather than a true increase in susceptibility to the infection.

An alternative hypothesis is that the differences in COVID-19 risk observed between ACE inhibitors and ARBs is due to residual confounding. We did not for example have data on ethnicity or socioeconomic status and it is possible that patients from different ethnic groups or social classes could be prescribed antihypertensives differentially and have different patterns of health-seeking behaviour.

Nevertheless, there remains the possibility that the higher risk of COVID-19 observed among users of ARBs is causal and that this class of drugs increases susceptibility to SARS-CoV-2 but not all-cause mortality. Further research is needed to test the biological plausibility and causality of this apparent effect. Further research is also needed to assess whether the use of ACE inhibitors and ARBs is associated with COVID-19 hospitalisation and death and whether any such associations differ between drug classes.

\section{Conclusions}

Prescription of ACE inhibitors was not associated with the risk of suspected or confirmed COVID-19 in primary care. By contrast, prescription of angiotensin II receptor blockers was associated with a statistically nonsignificant increase in risk. However, neither drug class was associated with all-cause mortality during the first peak of the pandemic. These findings need to be confirmed in other observational studies, potential pathways modelled through causal inference studies, and the basic mechanistic science of this potential association to be understood before recommendations can be made on the clinical implications for RAS inhibitor use during the ongoing COVID-19 pandemic. 


\section{Abbreviations}

ACE: Angiotensin-converting enzyme l; ARB: Angiotensin II type-1 receptor blockers; ARDS: Acute respiratory distress syndrome; BNF: British National Formulary; CCB: Calcium channel blocker; Cl: Confidence interval; COPD: Chronic obstructive pulmonary disease; COVID-19: Coronavirus disease-19; eGFR: estimated glomerular filtration rate; HR: Hazard ratio; ICU: Intensive care unit; NHS: National Health Service; OR: Odds ratio; RAS: Renin angiotensin system; RT-PCR: Reverse transcriptase polymerase chain reaction; SARS CoV-2: Severe acute respiratory syndrome coronavirus 2; SRC: Scientific review committee; THIN: The Health Improvement Network

\section{Supplementary Information}

The online version contains supplementary material available at https://doi. org/10.1186/s12879-021-05951-w.

Additional file 1: Supplementary Table 1. Read codes used for the ascertainment of the primary outcome (confirmed/suspected COVID-19)

Additional file 2: Supplementary Table 2. The RECORD statement for pharmacoepidemiology (RECORD-PE) checklist of items, extended from the STROBE and RECORD statements, which should be reported in noninterventional pharmacoepidemiological studies using routinely collected health data

\section{Acknowledgements}

Not applicable.

\section{Authors' contributions}

SH and KN conceived the research question and the study concept. NB, SD and DAM supported data provision. KG was responsible for data management and extraction. AS conducted the analysis with input from $T$, KN, JW and SH. JC and AA performed the literature review. KO, JSC, CS, NJA and DTZ assisted with development of clinical code lists. SH completed the first draft of the manuscript with input from KN, AS, JC and AA. All authors, including GNT, DP, TM, ES and NJA, reviewed and updated subsequent drafts and approved the final version.

\section{Funding}

This study was not externally funded.

\section{Availability of data and materials}

The data that support the findings of this study are available from The Health Improvement Network (THIN). Restrictions apply to the availability of these data, which were used under license for this study. Data are available from the authors with the permission of The Health Improvement Network $(T H I N)$.

\section{Declarations}

\section{Ethics approval and consent to participate}

The THIN data collection scheme and research carried out using THIN data were approved by the NHS South-East Multicentre Research Ethic Committee in 2003. Under the terms of the approval, studies must undergo independent scientific review. Approval for this study was obtained from the THIN Scientific Review Committee in June 2020 (SRC protocol reference 20-003-R2). Following approval, members of the research team were authorised to access the raw data for this study. All data were anonymised prior to use. The study protocol can be accessed at the European Union electronic Register of Post-Authorisation Studies (EU PAS) register (http://www.encepp. eu/encepp/viewResource.htm?id=35329).

\section{Consent for publication}

Not applicable.

\section{Competing interests}

The authors have no competing interests to declare. ES reports receiving funding from HDR-UK (PIONEER Hub), Wellcome, MRC, British Lung Foundation and NIHR. DP reports receiving funding from NIHR and MRC.

\section{Author details}

'Institute of Applied Health Research, University of Birmingham, Birmingham, UK. ${ }^{2}$ Cegedim Health Data, Cegedim Rx, London, UK. ${ }^{3}$ The Health Improvement Network (THIN), London, UK. ${ }^{4}$ Department of Diabetes, Gartnavel General Hospital, NHS Greater Glasgow and Clyde, Glasglow, UK. ${ }^{5}$ Birmingham Acute Care Research Group, Institute of Inflammation and Ageing, University of Birmingham, Birmingham, UK. ${ }^{6}$ Department of Acute Medicine, Queen Elizabeth Hospital Birmingham, Birmingham, UK. ${ }^{7}$ PIONEER, The Health Data Research UK Hub in Acute Care, Birmingham, UK. ${ }^{8}$ Department of Diabetes and Endocrinology, University Hospitals Birmingham NHS Foundation Trust, Birmingham, UK. ${ }^{9}$ Midlands Health Data Research UK, Birmingham, UK.

Received: 29 October 2020 Accepted: 2 March 2021

Published online: 15 March 2021

\section{References}

1. Williamson EJ, Walker AJ, Bhaskaran K, Bacon S, Bates C, Morton CE, et al. OpenSAFELY: factors associated with COVID-19 death in 17 million patients. Nature 2020:1-11. [cited 2020 Jul 9]; Nature Publishing Group. Available from: http://www.nature.com/articles/s41586-020-2521-4

2. COVID-19 Map - Johns Hopkins Coronavirus Resource Center. [cited 2020 Jul 7]. Available from: https://coronavirus.jhu.edu/map.html

3. Guan W, Ni Z, Hu Y, Liang W, Ou C, He J, et al. Clinical characteristics of coronavirus disease 2019 in China. N Engl J Med. 2020; Massachusetts Medical Society [cited 2020 Mar 28]. Available from: https://doi.org/10.1056/ NEJMoa2002032.

4. Zhang J, Dong X, Cao Y, Yuan Y, Yang Y, Yan Y, et al. Clinical characteristics of 140 patients infected with SARS-CoV-2 in Wuhan, China. Allergy Eur J Allergy Clin Immunol 2020;75(7):1730-1741. Blackwell Publishing Ltd; [cited 2020 Jul 30]. Available from: https://doi.org/10.1111/all.14238

5. Zhou F, Yu T, Du R, Fan G, Liu Y, Liu Z, et al. Clinical course and risk factors for mortality of adult inpatients with COVID-19 in Wuhan, China: a retrospective cohort study. Lancet. 2020;395(10229):1054-62. Lancet Publishing Group; [cited 2020 Jul 30]. https://doi.org/10.1016/S0140-6736(2 0)30566-3.

6. Patel $A B$, Verma A. Covid-19 and angiotensin-converting enzyme inhibitors and angiotensin receptor blockers: what is the evidence? JAMA. 2020; [cited 2020 Mar 26]; Available from: http://www.ncbi.nlm.nih.gov/pubmed/32208485.

7. Kuba K, Imai Y, Rao S, Gao H, Guo F, Guan B, et al. A crucial role of angiotensin converting enzyme 2 (ACE2) in SARS coronavirus-induced lung injury. Nat Med. 2005;11(8):875-9 Nature Publishing Group; [cited 2020 July 30] Available from: http://www.nature.com/naturemedicine.

8. Ozono R, Wang ZQ, Moore AF, Inagami T, Siragy HM, Carey RM. Expression of the Subtype 2 Angiotensin (AT 2 ) Receptor Protein in Rat Kidney. Hypertension 1997;30(5):1238-1246. Lippincott Williams and Wilkins; [cited 2020 July 30] Available from: https://doi.org/10.1161/01.HYP.30.5.1238

9. Wan Y, Shang J, Graham R, Baric RS, Li F. Receptor Recognition by the Novel Coronavirus from Wuhan: an Analysis Based on Decade-Long Structural Studies of SARS Coronavirus. J Virol. 2020;94(7):127-47 American Society for Microbiology; [cited 2020 July 30] Available from: http://jvi.asm.org/.

10. Kai H, Kai M. Interactions of coronaviruses with ACE2, angiotensin II, and RAS inhibitors-lessons from available evidence and insights into COVID-19. Hypertension Res 2020;43:648-654. Springer Nature; [cited 2020 Jul 21]. Available from: https://doi.org/10.1038/s41440-020-0455-8.

11. Kuba K, Imai Y, Penninger JM. Angiotensin-converting enzyme 2 in lung diseases. Curr Opin Pharmacol. 2006;6:271-6 Elsevier; [cited 2020 July 30]. Available from: /pmc/articles/PMC7106490/?report=abstract.

12. Position Statement of the ESC Council on Hypertension on ACE-Inhibitors and Angiotensin Receptor Blockers. [cited 2020 July 30]. Available from: https://www.escardio.org/Councils/Council-on-Hypertension-(CHT)/News/ position-statement-of-the-esc-council-on-hypertension-on-ace-inhibitors-a nd-ang

13. Aronson JK, Ferner RE. Angiotensin converting enzyme (ACE) inhibitors and angiotensin receptor blockers in COVID-19 - CEBM. [cited 2020 Jul 30]. Available from: https://www.cebm.net/covid-19/angiotensin-convertingenzyme-ace-inhibitors-and-angiotensin-receptor-blockers-in-covid-19/

14. Psaty BM, Koepsell TD, Lin D, Weiss NS, Siscovick DS, Rosendaal FR, et al. Assessment and control for confounding by indication in observational studies. J Am Geriatr Soc 1999;47(6):749-754. [cited 2020 July 30] Lippincott 
Williams and Wilkins; Available from: https://doi.org/10.1111/j.1532-5415.1 999.tb01603.x

15. Mancia G, Rea F, Ludergnani M, Apolone G, Corrao G. Renin-AngiotensinAldosterone System Blockers and the Risk of Covid-19. N Engl J Med 2020 [cited 2020 May 3]. Available from: https://doi.org/10.1056/NEJMoa2006923

16. Fosbøl EL, Butt JH, Østergaard L, Andersson C, Selmer C, Kragholm K, et al. Association of angiotensin-converting enzyme inhibitor or angiotensin receptor blocker use with covid-19 diagnosis and mortality. JAMA. 2020; [cited 2020 Jun 22]; Available from: http://www.ncbi.nlm.nih.gov/ pubmed/32558877.

17. COVID-19 Next steps in primary care surveillance. [cited 2021 Feb 4]. Available from: https://www.rcgp.org.uk/clinical-and-research/about/clinicalnews/2020/march/covid-19-next-steps-in-primary-care-surveillance.aspx

18. Hull SA, Williams C, Ashworth M, Carvalho C, Boomla K. Prevalence of suspected COVID-19 infection in patients from ethnic minority populations: A cross-sectional study in primary care. Br J Gen Pract 2020;70(699):E696E704. Royal College of General Practitioners; [cited 2021 Feb 4]. Available from: https://doi.org/10.3399/bjgp20X712601.

19. Lipsitch M, Tchetgen Tchetgen E, Cohen T. Negative Controls: A tool for detecting confounding and bias in observational studies. Epidemiology. 2010;21(3):383-8 NIH Public Access; [cited 2020 July 30]. Available from: /pmc/articles/PMC3053408/?report=abstract.

20. Health Survey for England 2018: Adult health. 2019 [cited 2020 July 30]. Available from: https://www.statisticsauthority.gov.uk/code-of-practice/

21. Hoffmann M, Kleine-Weber H, Schroeder S, Krüger N, Herrler T, Erichsen S, et al. SARS-CoV-2 Cell Entry Depends on ACE2 and TMPRSS2 and Is Blocked by a Clinically Proven Protease Inhibitor. Cell. 2020;181(2):271-280.e8 Cell Press; [cited 2020 July 30]. Available from:/pmc/articles/PMC7102627/ ?report=abstract.

22. Ferrario CM, Jessup J, Gallagher PE, Averill DB, Brosnihan KB, Tallant EA, et al. Effects of renin-angiotensin system blockade on renal angiotensin-(1-7) forming enzymes and receptors: Kidney International; Elsivier; 2005. p. 218996. [cited 2020 July 30]. Available from: http://www.kidney-international. org/article/S0085253815511150/fulltext

23. Ocaranza MP, Godoy I, Jalil JE, Varas M, Collantes P, Pinto M, et al. Enalapril attenuates downregulation of Angiotensin-converting enzyme 2 in the late phase of ventricular dysfunction in myocardial infarcted rat. Hypertens. 2006;48(4):572-8 Lippincott Williams \& Wilkins; [cited 2020 July 30]. Available from: http://www.ncbi.nlm.nih.gov/pubmed/16908757.

24. Sriram K, Insel PA. Risks of ACE Inhibitor and ARB Usage in COVID-19: Evaluating the Evidence. Clin Pharmacol Ther 2020;108(2):236-241. Nature Publishing Group; [cited 2020 July 30]. Available from: https://doi.org/10.1 002/cpt.1863

25. Liu Y, Yang Y, Zhang C, Huang F, Wang F, Yuan J, et al. Clinical and biochemical indexes from 2019-nCoV infected patients linked to viral loads and lung injury. Sci China Life Sci. 2020;63(3):364-74 Science in China Press; [cited 2020 July 30]. Available from:/pmc/articles/PMC7088566/?report= abstract.

26. Ye R, Liu Z. ACE2 exhibits protective effects against LPS-induced acute lung injury in mice by inhibiting the LPS-TLR4 pathway. Exp Mol Pathol. 2020; 113:104350 Academic Press Inc.

27. Yang G, Yang G, Tan Z, Tan Z, Zhou L, Yang M, et al. Effects of angiotensin II receptor blockers and ACE (angiotensin-converting enzyme) inhibitors on virus infection, inflammatory status, and clinical outcomes in patients with COVID-19 and hypertension: A single-center retrospective study. Hypertension 2020;76(1): 51-58. Lippincott Williams and Wilkins; [cited 2020 July 30]; Available from: https://doi.org/10.1161/HYPERTENSIONAHA.120.15143

28. Meng J, Xiao G, Zhang J, He X, Ou M, Bi J, et al. Renin-angiotensin system inhibitors improve the clinical outcomes of COVID-19 patients with hypertension. Emerging Microbes Infect 2020;9:757-760. Taylor and Francis Ltd.; [cited 2020 July 30]. Available from: https://doi.org/10.1080/22221751.2 020.1746200

29. Grover A, Oberoi M. A systematic review and meta-analysis to evaluate the clinical outcomes in COVID -19 patients on angiotensin converting enzyme inhibitors or angiotensin receptor blockers. medRxiv. 2020;2020(04):29. 20085787 Cold Spring Harbor Laboratory Press.

30. Pranata R, Permana H, Huang I, Lim MA, Soetedjo NNM, Supriyadi R, et al. The use of renin angiotensin system inhibitor on mortality in patients with coronavirus disease 2019 (COVID-19): A systematic review and meta-analysis. Diabetes Metab Syndr Clin Res Rev. 2020;14(5):983-90 Elsevier Ltd; [cited 2020 July 30]. Available from: /pmc/articles/PMC7319940/?report=abstract.
31. Abdulhak AA, Kashour T, Noman A, Tlayjeh H, Mohsen A, Al-Mallah MH, et al. Angiotensin converting enzyme inhibitors and angiotensin receptor blockers and outcome of covid-19: a systematic review and meta-analysis. medRxiv. 2020 Cold Spring Harbor Laboratory Press; [cited 2020 Aug 3]. Available from: https://doi.org/10.1101/2020.05.06.20093260

32. GHOSAL S, Mukherjee JJ, Sinha B, Gangopadhyay KK. The effect of angiotensin converting enzyme inhibitors and angiotensin receptor blockers on death and severity of disease in patients with coronavirus disease 2019 (COVID-19): a meta-analysis. medRxiv 2020. Cold Spring Harbor Laboratory Press; [cited 2020 Aug 3]. Available from: https://doi.org/10.11 01/2020.04.23.20076661.

33. Zhang $X$, Yu J, Pan L, Jiang H. ACEI/ARB use and risk of infection or severity or mortality of COVID-19: A systematic review and meta-analysis. Pharmacol Res. 2020;158:104927 Academic Press; [cited 2020 Sep 1]. Available from: /pmc/articles/PMC7227582/?report=abstract.

34. Morales DR, Areia C, Krumholz HM, Prieto-Alhambra D, Ryan PB, Hripcsak G, et al. Renin-angiotensin system blockers and susceptibility to COVID-19: a multinational open science cohort study. medRxiv 2020;20:21. [cited 2020 June 15]. Available from: https://doi.org/10.1101/2020.06.11.20125849.

35. Hippisley-Cox J, Young D, Coupland C, Channon KM, Tan PS, Harrison DA, et al. Risk of severe COVID-19 disease with ACE inhibitors and angiotensin receptor blockers: cohort study including 8.3 million people. Heart 2020; [cited 2020 Aug 4]. Available from: https://doi.org/10.1136/heartjnl-2020-317393

36. Dicpinigaitis PV. Angiotensin-converting enzyme inhibitor-induced cough accp evidence-based clinical practice guidelines. Chest. 2006;129 [cited 2020 June 11]. Available from: www.chestjournal.org.

\section{Publisher's Note}

Springer Nature remains neutral with regard to jurisdictional claims in published maps and institutional affiliations.

Ready to submit your research? Choose BMC and benefit from:

- fast, convenient online submission

- thorough peer review by experienced researchers in your field

- rapid publication on acceptance

- support for research data, including large and complex data types

- gold Open Access which fosters wider collaboration and increased citations

- maximum visibility for your research: over $100 \mathrm{M}$ website views per year

At BMC, research is always in progress.

Learn more biomedcentral.com/submissions 